\title{
La satisfaction des patients et de leur famille vis-à-vis de l'information relative au cancer : une recension des écrits
}

\author{
Par Kim Chapman et Kathy Rush
}

\begin{abstract}
Abrégé
Les individus et les familles qui font face à l'expérience du cancer ont des besoins d'apprentissage à la fois nombreux et variés. L'enseignement relatif au cancer constitue un aspect important de leurs soins. Pourtant, au cours des trois dernières décennies, il est régulièrement question, dans la littérature, de l'insatisfaction d'un grand nombre de patients et de proches vis-à-vis de l'enseignement qui leur est fourni dans ce domaine. Cet article examine les écrits relatifs à un aspect de la satisfaction vis-à-vis de l'enseignement lié au cancer et à la satisfaction des patients et des proches vis-à-vis de l'information liée au cancer, et ce, en fonction de facteurs liés aux patients, aux prestataires et à l'information. Les lacunes au niveau des recherches sont dégagées et discutées dans le cadre des recommandations pour de futurs travaux de recherche. Cet article se termine par des applications à la pratique clinique.
\end{abstract}

L'information est considérée comme étant importante pour toute personne aux prises avec un problème de santé, mais pour les individus et les familles touchés par le cancer, elle est essentielle. Les personnes atteintes du cancer et leur famille ont tendance à vouloir le plus d'information possible au sujet du diagnostic, du traitement et des autres aspects des soins aux personnes atteintes du cancer, que cette information soit bonne ou mauvaise (Cassileth, Zupkis, SuttonSmith et March, 1980; Jones et al., 1999; Tattersall, Butow, Griffin et Dunn, 1994; Turner, Maher, Young, Young et Hudson, 1996). Tout au long de l'expérience du cancer, les besoins d'informations et de compétences nouvelles sont considérables. Les patients et leurs proches doivent s'adapter aux diverses phases de l'expérience, à l'omniprésence de la maladie et aux besoins continuels du malade, lesquels sont susceptibles d'évoluer rapidement.

Il existe un imposant corpus de recherche axé sur la satisfaction des patients vis-à-vis des soins aux personnes atteintes du cancer en général. Cependant, on a accordé moins d'attention à leur satisfaction vis-à-vis de l'enseignement aux patients. Pourtant, Sitzia et Wood (1998a) ont identifié l'enseignement aux patients comme l'une des quatre composantes de la satisfaction des patients atteints de cancer traités en clinique externe. Bien que l'on reconnaisse l'importance des échanges d'information au sein du processus éducationnel, la satisfaction des patients vis-à-vis de l'information qu'ils reçoivent reste grandement inexplorée.

L'insatisfaction des patients vis-à-vis de la diffusion de l'information remonte aux années 1970 (Risser, 1975). Les données accumulées depuis cette époque indiquent que les patients atteints du cancer, quel que soit le type de cancer ou quelle que soit la phase de la maladie, sont souvent insatisfaits de l'information qui leur est communiquée (Northouse, Schafer, Tipton et Metivier, 1999; Sitzia et Wood, 1998a, 1998b). L'insatisfaction générale rehausse l'importance de comprendre les facteurs qui influent sur la satisfaction des patients vis-à-vis de la communication de l'information (Cassileth et al., 1980; Derdiarian, 1989; Fitch, Johnson, Gray et Franssen, 1999).
Le but de cet article est d'examiner les recherches liées à la satisfaction des patients et de leurs proches vis-à-vis de l'information relative au cancer qui leur est communiquée. Nous présenterons une synthèse et une analyse des écrits de recherche disponibles dans ce domaine. En conclusion, nous soulignerons les lacunes à l'intérieur des études réalisées à ce jour et présenterons des suggestions pour la pratique clinique et pour de futurs projets de recherche.

\section{Les facteurs qui influent sur la satisfaction des patients et de leurs proches vis-à-vis de l'information relative au cancer}

Nous avons utilisé les bases de données MEDLINE et CINAHL dans le cadre de cette étude documentaire en raison de leur étendue et de leur capacité à cibler la recherche sur la satisfaction des patients atteints du cancer et de leur famille vis-à-vis de l'information. Nous avons sélectionné des articles de recherche allant du début des années 1970 jusqu'au mois d'août 2002 afin d'inclure toutes les études axées sur la satisfaction des patients et de leurs familles vis-à-vis de l'information relative au cancer. Les mots clés employés dans la recherche comprenaient: satisfaction, attentes, perceptions, enseignement $\mathrm{au}(\mathrm{x})$ patient(s), enseignement, cancer, information relative au cancer et oncologie. Parmi tous les écrits sur la satisfaction des patients passés en revue, environ le quart était axé sur les facteurs déterminants, du point de vue des patients, de la satisfaction des patients vis-à-vis des soins aux personnes atteintes du cancer. Certaines études se fondaient sur des cas cliniques isolés tandis que d'autres étaient fondées sur la recherche. Nous avons recueilli des articles de recherche additionnels en examinant manuellement les références bibliographiques des articles utilisés dans le cadre de notre étude. Pour les fins de cette étude, seuls les articles de nature empirique fondés sur la recherche ont été retenus. Nous avons inclus dans l'étude des articles classiques et des travaux plus récents. Les articles ont été sélectionnés sans tenir compte de leur définition du terme "satisfaction". À partir des documents empiriques étudiés, les auteures (K et KR) ont identifié trois catégories qui reflètent les principales influences sur la satisfaction des patients vis-à-vis de l'information relative au cancer: 1) les facteurs liés aux patients, 2) les facteurs liés aux fournisseurs d'information, et 3 ) les facteurs liés à l'information elle-même. Ces catégories interdépendantes ont servi de cadre organisationnel et de guide au cours de la réalisation de l'étude documentaire, et aucune des catégories n'a été perçue comme étant plus importante que les autres.

Kim Chapman, $R N, \operatorname{MSc}(N)$, est infirmière clinicienne spécialisée, Oncologie, River Valley Health, Fredericton, NB.

Kathy Rush, RN, PhD, est professeure à la University of South Carolina, Mary Black School of Nursing, Spartanburg, SC. Lors de la rédaction de cet article, Kathy Rush était professeure à l'Université du Nouveau-Brunswick à Fredericton, NouveauBrunswick. 


\section{Les facteurs liés aux patients et à leurs proches}

La recherche indique que les facteurs liés aux patients et aux proches constituent d'importants déterminants de la satisfaction visà-vis de l'information. Nous avons identifié une gamme de facteurs liés aux patients et à leurs proches qui semblent influer sur la satisfaction. Ces facteurs comprennent les attentes, les préférences relatives au type d'information, le style de recherche d'information, l'anxiété, les données démographiques et l'observance du traitement.

\section{Les attentes}

Les études portant sur la satisfaction générale des patients semblent indiquer que les attentes influencent la satisfaction des patients vis-à-vis des soins, et ce parmi les patients traités dans des services internes médicaux-chirurgicaux et gynécologiques, de même que dans des unités spécialisées (Abramowitz, Coté et Berry, 1987; Green, Coupland et Kitzinger, 1990). La nature de l'interaction entre les attentes et la satisfaction des patients atteints du cancer est mal cernée puisque les études portant sur cette interaction sont peu nombreuses. Nous en savons encore moins sur les attentes des personnes atteintes du cancer en matière d'information et sur l'impact de ces attentes sur la satisfaction. Dans son étude classique datant de 1984 sur les attentes des patients atteints du cancer vis-à-vis de l'ensemble des soins et sur leur satisfaction vis-à-vis de l'information, Oberst (1984) a rapporté une corrélation positive entre les attentes des patients atteints du cancer $(n=20)$ qui recevaient un traitement ambulatoire de chimiothérapie et leur satisfaction vis-à-vis des soins auto-administrés $(\mathrm{r}=0,626)$ et de l'information relative au diagnostic et au traitement $(\mathrm{r}=0,685)$. En 1988, Degner et Russell ont rapporté une corrélation positive entre la satisfaction des patients $(n=60)$ vis-àvis des soins de santé et la concrétisation des attentes en matière d'information. Les travaux de recherche montraient que le degré d'exhaustivité de l'information exerce une influence importante sur la mesure dans laquelle les attentes sont satisfaites en matière d'information, ce qui semble indiquer l'existence d'une corrélation entre le caractère adéquat de l'information et la satisfaction générale vis-à-vis des soins (Oberst). Malgré le rôle central des attentes dans la compréhension de la satisfaction des patients atteints du cancer vis-àvis des soins, nous n'avons pas pu trouver de recherches plus poussées basées sur ces travaux fondateurs.

\section{Les préférences liées aux types d'information}

Les préférences des patients et des proches pour certains types d'information relative au cancer constituent une variable importante et déterminante, bien que mal comprise, dans la satisfaction vis-à-vis de l'information (Degner et Russell, 1988). Une des raisons pour lesquelles cette variable est mal comprise est la gamme de significations différentes du terme "préférences des patients" que l'on retrouve dans les écrits. Ce terme apparaît de façon explicite dans certaines études, tandis que d'autres travaux utilisent des termes analogues tels que "besoins d'apprentissage", "perception du caractère adéquat" et "utilité de l'information".

Plusieurs études ont dégagé, chez les patients, une gamme de préférences pour certains types d'information tels que la maladie, les examens, les options de traitement, les résultats anticipés, à quoi s'attendre et quoi faire lors d'un événement menaçant et le soulagement des symptômes (Cassileth, et. al., 1980; Derdiarian, 1986, 1987, 1989; Kutner, Foehner et Steiner, 1999; Northouse et al., 1999; Sutherland, Llwellyn-Thomas, Lockwood, Tritchler et Till, 1989). Les résultats concernant la relation entre les préférences en matière d'information et la satisfaction des patients ne sont pas concluants. Par exemple, parmi un échantillon d'hommes récemment diagnostiqués d'un cancer et de leurs épouses, on a observé une corrélation positive entre la satisfaction des patients et de leur famille et le type et la quantité d'information fournis, et ce dans chacune des quatre catégories d'information suivantes: relative à la maladie, de nature personnelle, relative à la famille et de nature sociale $(\mathrm{p}<0,01)$ (Derdiarian, 1989). Par contre, une étude pancanadienne plus récente portant sur les expériences vécues par des hommes atteints du cancer de la prostate $(n=621)$ indique que presque $20 \%$ des hommes se sont dit insatisfaits de l'information qu'ils avaient reçue au sujet des réactions émotionnelles (19\%), des médecines non conventionnelles et parallèles $(18,5 \%)$, de ce qu'il faut faire pour parler à d'autres hommes atteints de ce même cancer $(17,4 \%)$, des services de counseling disponibles, des groupes d'entraide et des groupes de soutien $(15,1 \%)$ (Fitch et al., 1999).

Dans la plupart des études portant sur les préférences des patients et des proches, ceux-ci et ceux-là sont traités comme un seul groupe homogène, ce qui rend difficile la différentiation entre les préférences des patients et celles de leurs soignants naturels/proches. Bien que Northouse et collègues (1999) aient trouvé que les conjoints étaient toujours à l'affût de plus d'information au sujet de la maladie et du traitement, le degré de satisfaction particulier des conjoints vis-à-vis de l'information était occulté par l'utilisation d'un taux de satisfaction générale vis-à-vis de l'information. Des études récentes indiquent que les patients et leurs soignants naturels peuvent avoir des préférences différentes quant au type d'information relative au cancer (AucoinGallant, 1999; Rees et Bath, 2000). Nous n'avons trouvé qu'une seule étude qui tenait compte séparément des préférences des soignants naturels en matière d'information $(n=30)$ (Aucoin-Gallant). Dans l'étude d'Aucoin-Gallant, les soignants naturels souhaitaient, bien sûr, recevoir de l'information relative à la maladie et au traitement, mais ils classaient l'information de nature psychosociale parmi leurs trois plus importants besoins informationnels. En particulier, plus de $80 \%$ des soignants ont rapporté les besoins suivants: comment composer avec les comportements sociaux négatifs, l'impact psychologique du cancer et du traitement ainsi que la probabilité de rémission ou de récurrence. À la lumière des résultats d'AucoinGallant, il conviendrait que les études qui traitent les patients et leurs familles comme un seul groupe homogène lors de l'analyse des données envisagent l'étude de chaque élément du groupe d'étude séparément.

\section{Le style de recherche d'information}

Des études de recherche ont apporté un éclairage instructif sur la relation entre la satisfaction des patients et le style de recherche d'information (Burish et Tope, 1992; Miller, 1987). En ce qui concerne la population atteinte de cancer, Steptoe, Sutcliffe, Allen et Coombes (1991) ont observé au sein de leur échantillon de patients atteints d'un cancer métastatique $(n=77)$ que ceux qui évitaient toute information ou qui préféraient en recevoir le moins possible (les patients "non intéressés") avaient un taux plus élevé de satisfaction vis-à-vis de l'information relative au cancer, alors que ceux qui étaient activement à la recherche d'information (les patients "intéressés") avaient un niveau plus élevé de connaissances factuelles. Les résultats semblent indiquer que les patients non intéressés qui rapportaient une bonne compréhension de leur condition et manifestaient leur satisfaction vis-à-vis de l'information fournie étaient satisfaits non pas en raison de leur niveau élevé de connaissances factuelles, mais en raison de leur style d'adaptation.

\section{L'anxiété}

Toutes les études signalent de façon constante que l'anxiété est l'un des principaux prédicteurs de la satisfaction générale vis-à-vis des soins de santé, peu importe la population à l'étude (Oberst, 1984; Sitzia et Wood, 1998b; Steptoe et al., 1991; Waitzkin, 1984).

En ce qui concerne la population atteinte du cancer, quelques études ont rapporté des résultats semblables. Oberst a observé que l'anxiété des patients était le meilleur prédicteur $(\mathrm{p}<0,001)$ de la satisfaction générale au sein d'un échantillon de patients ambulatoires recevant un traitement de chimiothérapie $(n=20)$. Pour ce qui est de 
l'information liée au cancer, Oberst a de plus observé une faible corrélation entre l'anxiété et la satisfaction vis-à-vis du diagnostic ( $\mathrm{r}=$ $0,241)$ et de l'information relative aux autosoins $(r=-0,336)$. D'autres chercheurs ont également observé une corrélation significative entre l'anxiété et la satisfaction (Jones et al., 1999; Steptoe et al., 1991; Tattersall et al., 1994). Steptoe et ses collègues ont observé que les personnes qui se disaient très satisfaites de l'information relative au cancer avaient un niveau moins élevé d'anxiété réactionnelle $(\mathrm{p}<0,05)$. Les résultats de l'étude de Tattersall et collègues indiquent que le meilleur prédicteur de la satisfaction vis-à-vis des bandes sonores et des lettres d'information était l'anxiété du patient avant la consultation $(\mathrm{p}<0,0001)$. Ces chercheurs ont découvert que la satisfaction vis-à-vis de la bande sonore diminuait à mesure que l'anxiété augmentait.

\section{Les données démographiques}

Un certain nombre de caractéristiques démographiques en relation avec la satisfaction des patients vis-à-vis de l'information relative au cancer ont été étudiées. Les résultats ne sont pas concluants en ce qui concerne les effets de l'âge, du sexe, du niveau d'éducation, du statut socio-économique, des croyances religieuses et du type de traitement sur la satisfaction vis-à-vis de l'information relative au cancer (Butow, Maclean, Dunn, Tattersall et Boyer, 1997; Butow, Brindle, McConnell, Boakes et Tattersall, 1998; Cassileth et al., 1980; Derman et Serbest, 1993; Steptoe et al., 1991; Tattersall et al., 1994). L'effet confondant de l'étude simultanée de plusieurs variables démographiques est l'interprétation la plus plausible.

Les résultats de plusieurs autres études semblent indiquer une certaine corrélation entre les données démographiques des patients et la satisfaction vis-à-vis de l'information relative au cancer. On a constaté des résultats contradictoires sur la relation entre l'âge et la satisfaction (Butow et al., 1997; Ong et al., 2000; Sitzia et Wood, 1998b; Steptoe et al., 1991; Tattersall et al., 1994). Une étude récente randomisée à double insu auprès de patients atteints du cancer qui assistaient à leur première consultation clinique externe a révélé une interaction entre l'âge des patients et le mode de diffusion de l'information. L'étude a établi que cette interaction exerçait une influence sur la satisfaction des patients vis-à-vis de l'information (Ong et al.). Les patients plus jeunes étaient plus satisfaits que les participants plus âgés $(\mathrm{p}<0,01)$ lorsque le mode de diffusion de l'information consistait en une bande sonore. D'autres chercheurs (Jones et al., 1999) ont trouvé que les patients âgés de moins de 60 ans manifestaient plus d'insatisfaction vis-à-vis de l'information fournie (type et quantité) $(\mathrm{p}<0,05)$. Dans leur enquête, Jones et collaborateurs ont observé que les hommes étaient moins satisfaits de l'information fournie que les femmes $(p<0,05)$. Par contre, parmi des patients thailandais bouddhistes atteints du cancer et qui recevaient un traitement de radiothérapie, les hommes se sont montrés plus satisfaits que les femmes vis-à-vis de l'information fournie par les infirmières (Lundberg et Trichorb, 2001).

Bien que limités, les résultats indiquent qu'il se peut que le type de cancer et la situation de famille influent sur la satisfaction vis-à-vis de l'information relative au cancer. Les chercheurs ont observé que le type de cancer constitue une variable confusionnelle dans la corrélation entre le mode de diffusion de l'information et la satisfaction, ce qui semble indiquer une relation indirecte entre ces deux variables (Dunn et al., 1993; Tattersall et al., 1994). La seule étude qui tenait compte de la situation de famille révélait que les patients qui ne s'étaient jamais mariés étaient plus satisfaits de la bande sonore que ceux qui étaient mariés, divorcés ou veufs $(\mathrm{p}<0,01)$ (Tattersall et al.).

\section{L'observance du traitement}

La recherche a révélé l'existence d'une corrélation directe entre l'observance du traitement (la définition de son contraire étant l'interruption du traitement) et la satisfaction du patient vis-à-vis des soins de chimiothérapie (Sitzia et Wood, 1998b). Dans une étude comparative réalisée auprès de patients recevant un traitement de chimiothérapie, on a trouvé que les patients qui interrompaient leur traitement étaient moins satisfaits de l'ensemble des aspects des soins infirmiers, y compris l'information fournie aux patients $(\mathrm{p}<0,01)$. Plus particulièrement, des analyses plus approfondies de facettes particulières de l'information ont révélé que les patients qui interrompaient leur traitement étaient considérablement moins satisfaits de l'information relative aux aspects pratiques de la gestion des effets secondaires $(\mathrm{p}<0,01)$. Des études antérieures avaient souligné le fait que ce sont les difficultés liées à la gestion des effets secondaires qui contribuent à l'interruption du traitement, et non pas la seule présence de ces derniers (Richardson, Marks et Levine, 1988). Malgré le fait que les patients qui interrompent leur traitement semblent moins satisfaits de l'information, nous ne savons pas si l'observance du traitement est un facteur lié au patient ou un résultat de la satisfaction vis-à-vis de l'information.

\section{Facteurs liés aux fournisseurs de l'information}

On a observé que les caractéristiques des fournisseurs influent sur la satisfaction des patients et de leurs proches vis-à-vis de l'information fournie. Un corpus de recherche spécifique décrit toute une gamme de facteurs liés aux fournisseurs qui influent sur la satisfaction des patients et de leur famille vis-à-vis de l'information relative au cancer. On a identifié des facteurs généraux tels que la fonction du fournisseur de l'information, de même que des facteurs très spécifiques tels que les caractéristiques particulières dudit fournisseur.

\section{Les fournisseurs de l'information}

Quelques études, surtout descriptives, ont rapporté que la fonction $\mathrm{du}$ fournisseur de l'information semble jouer un rôle dans la satisfaction des patients. Des études plus anciennes ont rapporté que les patients atteints du cancer préféraient recevoir l'information de leur médecin, et ne percevaient pas les infirmières comme étant des sources valides d'information (Oberst, 1984; Tishelman, 1994). Cependant, on observe le contraire dans des travaux empiriques plus récents. Une étude effectuée auprès de patients britanniques qui recevaient des soins en clinique externe rapportait que $85 \%$ des patients étaient "toujours" satisfaits de l'information fournie par les infirmières, alors que $47 \%$ des patients se disaient satisfaits des explications des médecins (Hutchison, Addington-Hall, Bower, Austen et Coombes, 1991). Sitzia et Wood (1998b) ont également rapporté des taux de satisfaction plus élevés vis-à-vis de l'information lorsque celle-ci était fournie par les infirmières que lorsqu'elle était transmise par les médecins.

\section{Le style de communication}

La recherche a identifié le style de communication comme étant une variable importante dans la détermination de la satisfaction des patients (Sitzia et Wood, 1998a). La méthode employée pour communiquer l'information dépend en partie de l'accessibilité du fournisseur de l'information. La comparaison des études est compliquée par le fait que chacune d'entre elles emploie une définition différente du terme "accessibilité". Pienschke (1973) définit l'accessibilité comme une disposition à fournir de l'information et à ne pas la retenir. Elle a observé des taux de satisfaction plus élevés parmi les patients lorsque le fournisseur adoptait une approche ouverte qui permettait d'informer le patient de façon explicite au sujet des détails du diagnostic et du pronostic. Deux autres études ont examiné l'accessibilité du fournisseur (Kutner et al., 1999; Sitzia et Wood, 1998b). Sitzia et Wood (1998b) ont défini l'accessibilité comme l'ensemble des caractéristiques interpersonnelles du fournisseur qui incitent les patients à poser des questions. Ils ont observé une forte corrélation positive entre l'accessibilité du consultant et la satisfaction vis-à-vis de l'information relative au diagnostic $(\mathrm{p}<0,001)$. Pour leur part, Kutner et associés ont défini l'accessibilité en termes de style de prise de décisions. Dans leur étude, les patients ont rapporté des taux 
de satisfaction plus élevés vis-à-vis de l'information fournie par les médecins lorsque ceux-ci adoptaient un style de prise de décisions participatif.

Il nous a été difficile d'isoler la relation spécifique entre le style de communication et la satisfaction des patients vis-à-vis de l'information parce que les facteurs liés aux patients semblent jouer un rôle d'intermédiaires. Lorsque Steptoe et collègues (1991) ont interviewé des malades hospitalisés dans une unité d'oncologie médicale, ils ont observé des taux de satisfaction vis-à-vis de la communication plus élevés parmi les patients qui avaient pourtant de moindres connaissances factuelles relatives au cancer. Cependant, les taux de satisfaction vis-à-vis des soins ne semblent pas être modifiés lorsqu'on fournit de l'information de façon à en faciliter la rétention (Ley, 1982; Reynolds, Byrne, Sanson-Fisher, Poole et Harker, 1981).

\section{La conduite et les réactions du fournisseur}

La recherche a révélé qu'il existe une corrélation entre l'anxiété du fournisseur et la satisfaction des patients (Carter, Inui, Kukull et Haigh, 1982; Hall, Roter et Rand, 1981; Waitzkin, 1984). Au début des années 1980, Carter et collègues ont trouvé que le taux de satisfaction des patients nouvellement diagnostiqués d'un cancer augmentait lorsque le ton de voix du médecin trahissait de l'anxiété. Quelques années plus tard, Waitzkin a avancé que les patients étaient plus satisfaits des fournisseurs qui manifestaient de l'anxiété lorsqu'ils leur offraient de l'information.

Selon Richardson et ses collègues (1988), les commentaires des patients indiquaient que leurs perceptions des efforts des infirmières pour les aider à gérer les effets secondaires influaient sur le niveau de satisfaction des patients, que les résultats en matière d'effets secondaires soient éventuellement positifs ou négatifs. De plus, Poroch (1995) a postulé que l'emploi de descriptions sensorielles et procédurales de la part de l'infirmière donnait aux patients l'impression que cette dernière comprenait bien l'expérience qu'ils étaient sur le point de vivre et qu'elle en avait une compréhension empathique.

\section{Facteurs liés à l'information}

Les études ont permis d'identifier des éléments tels que le mode de communication de l'information, le degré de personnalisation de l'information, la quantité d'information et le moment choisi pour communiquer l'information comme étant des variables qui influencent la satisfaction vis-à-vis de l'information relative au cancer (Poroch, 1995; van Wersch et al., 1997). Étant donné que les facteurs liés à l'information sont intégralement associés aux facteurs liés aux patients et aux fournisseurs de l'information, certains aspects des facteurs liés à l'information ont déjà été abordés dans les sections cidessus. Cette section soulignera les aspects spécifiques des facteurs liés à l'information qui influent sur la satisfaction des patients.

\section{Le mode de communication de l'information}

Bien qu'elle comporte des limites, la recherche indique que le mode de communication de l'information constitue une importante variable de la satisfaction des patients atteints du cancer vis-à-vis de l'information qu'ils reçoivent. Les chercheurs ont examiné divers modes de communication, dont le registre, les livrets et feuillets d'information et les cassettes audio (Dunn et al., 1993; Hagopian, 1996; Tattersall et al., 1994; Vetto, Dubois et Perez Vetto, 1996; Whelan et al., 1998).

L'utilisation de livrets et feuillets d'information est très répandue dans la transmission de l'information relative au cancer. Les études sur la satisfaction des patients vis-à-vis de ces modes de communication très courants se sont soldées par des résultats variables. La distribution de feuillets d'information à 108 femmes atteintes d'une affection bénigne du sein s'est traduite par un niveau élevé de satisfaction générale $(91 \%)$. Dans une autre étude, la distribution d'une chemise contenant des livrets et feuillets d'information aux patients nouvellement diagnostiqués d'un cancer a permis d'accroître la satisfaction de façon significative ( $p<0,0001)$ (Whelan et al., 1998). Cependant, les patients qui recevaient leur premier traitement de chimiothérapie n'étaient pas plus satisfaits de l'utilisation combinée d'un manuel et d'une séance d'enseignement au patient personnalisée d'une durée de 15 minutes offerte par une infirmière que du programme didactique standard d'une durée de 45 minutes (Porter, 1998). Parmi les études que nous avons passées en revue, la seule qui examinait la satisfaction des patients en fonction des caractéristiques des livrets et feuillets a révélé que les patients accordaient la même cote élevée à cinq livrets sur le cancer dont le contenu, les idées maîtresses et l'origine variaient, sans qu'ils aient constaté une différence quelconque entre les ouvrages (Butow et al., 1998).

Des travaux de recherche indiquent que l'utilisation de bandes sonores semble contribuer à l'augmentation de la satisfaction des patients atteints du cancer (Dunn et al., 1993; Hagopian, 1996; Ong et al., 2000; Tattersall et al., 1994). Ong et ses collègues ont observé une plus grande satisfaction vis-à-vis de l'information parmi les patients plus jeunes qui ont reçu un enregistrement audio de leur consultation initiale que parmi ceux du groupe témoin qui, eux, n'ont pas reçu de tel enregistrement. On a observé une plus grande satisfaction parmi les patients qui écoutaient des bandes sonores présentant de l'information d'ordre général sur la radiothérapie et ses effets secondaires que parmi les patients du groupe témoin qui recevaient les soins standard sans bande sonore (Hagopian). En raison des difficultés liées à l'interprétation des scores de satisfaction tels que rapportés, les chercheurs n'ont pas pu conclure avec certitude que la satisfaction accrue était statistiquement significative. Des recherches montrent que l'information personnalisée enregistrée sur support audio pour chaque patient augmente encore davantage la satisfaction des patients (Dunn et al.). Par contre, les patients atteints du cancer qui assistaient à leur première consultation auprès d'un oncologue médical ne se disaient pas plus satisfaits de l'information transmise par bande sonore que par lettre de récapitulation (Tattersall et al.).

\section{L'information personnalisée par rapport à l'information générale}

Les différentes études consultées indiquent que la personnalisation de l'information selon la situation particulière et le type de cancer du patient augmente la satisfaction (Derdiarian, 1989; Dunn et al., 1993; Jones et al., 1999). Derdiarian (1989) a observé que les hommes récemment diagnostiqués d'un cancer et leur épouse à qui l'on avait offert un ensemble formel et personnalisé d'information, de counseling et d'orientation vers les ressources étaient considérablement plus satisfaits de l'information qu'ils avaient reçue que les membres du groupe témoin, à qui l'équipe professionnelle de la clinique avait offert un ensemble standard non formel et non personnalisé de counseling et d'orientation vers les ressources ou de soins de suivi. Dans une étude expérimentale sur échantillon contrôlé, Dunn et associés ont rapporté que la satisfaction des patients atteints du cancer qui assistaient à leur première consultation auprès d'un oncologue médical était considérablement plus élevée lorsque les patients recevaient un enregistrement audio d'une consultation personnalisée $(91 \%)$ que lorsqu'ils recevaient un enregistrement générique décrivant le cancer $(87,2 \%)$ ou lorsqu'ils ne recevaient pas de bande sonore du tout $(85 \%)(\mathrm{p}<0,05)$. Loin de renseigner les patients au sujet des faits relatifs à leur cas particulier, les bandes sonores contenant de l'information générale semblaient être pour les patients source de confusion ou de déplacement de l'attention (Dunn et al.). En utilisant un différent mode de communication (l'ordinateur), Jones et collègues ont rapporté des résultats semblables. Leur essai randomisé concernant l'information personnalisée fournie par ordinateur montre que les patients sont plus satisfaits lorsqu'ils reçoivent des renseignements personnalisés (différence moyenne: 12 $\%, 95 \%$ intervalle de confiance $0,7 \%-23,9 \%$ ).

Les patients que l'on préparait à la radiothérapie au moyen de programmes éducatifs structurés et personnalisés comprenant de l'information sensorielle et procédurale (groupe expérimental) étaient 
considérablement plus satisfaits de la quantité et de la spécificité de l'information que les patients préparés au moyen de la méthode hospitalière standard et non structurée (groupe témoin) $(\mathrm{p}=0,000)$ (Poroch, 1995). Ce taux de satisfaction demeurait inchangé dans le temps, à partir des quelques jours suivant le début de la radiothérapie jusqu'à la dernière semaine du traitement de chaque patient $(\mathrm{p}<0,001)$.

\section{La quantité d'information}

Les recherches n'ont pas clairement établi la relation entre la satisfaction des patients et la quantité d'information. Dans une étude de Turner et collègues (1996) qui a mis au jour l'insatisfaction des patients vis-à-vis de la quantité d'information, $90 \%$ des patients qui avaient reçu un traitement pour la maladie de Hodgkin et qui n'avaient pas reçu d'information écrite (62\%) auraient aimé recevoir du matériel écrit. Cependant, dans une autre étude, des femmes atteintes du cancer du sein qui avaient reçu une quantité "substantielle" d'information dans le cadre d'un essai clinique ont trouvé cette information inadéquate (Reynolds et al., 1981).

Les recherches semblent indiquer que la quantité d'information varie au cours de l'expérience du cancer, mais les résultats sont compliqués par le fait que les patients ont différentes préférences en matière d'information. Sitzia et Wood (1998b) ont rapporté une corrélation positive entre la satisfaction des patients et la quantité d'information reçue, mais ils ont également observé que le sujet de l'information demandée par les patients constituait manifestement une variable intermédiaire. Une autre étude a rapporté que le type d'information requise par les malades atteints du cancer qui en étaient à la phase du traitement clinique externe était stable dans le temps, mais que la quantité d'information réclamée diminuait entre la première et la deuxième consultation médicale (Butow et al., 1997).

\section{Le choix du moment}

Le choix du moment où communiquer l'information relative au cancer est de plus en plus reconnu comme étant un facteur qui influence la satisfaction des patients vis-à-vis de l'information. Nous n'avons trouvé qu'une seule étude comparative qui utilisait le choix du moment (séquence temporelle de la communication de l'information) comme variable indépendante dans la mesure de la satisfaction vis-àvis de l'information des patients recevant un traitement de radiothérapie (D'haese et al., 2000). Les patients qui ont reçu leur information par étapes (un livret avant la stimulation, suivi de feuillets d'information durant les troisième et quatrième jours de traitement) se sont dits considérablement plus satisfaits au cours de la dernière semaine de traitement que les patients qui avaient reçu à la fois le livret et les feuillets avant la stimulation (groupe simultané) $(\mathrm{p}=0,001)$.

\section{Lacunes dans la recherche à ce jour et indications pour la recherche à venir}

Jusqu'à présent, l'étude de la satisfaction vis-à-vis de l'information au sein de la population atteinte du cancer a été en grande partie non théorique. Bien que l'on ait utilisé la théorie du stress et de l'adaptation et la théorie des systèmes familiaux dans le cadre d'études générales des besoins d'information des patients atteints du cancer et de leurs proches, l'étude de la satisfaction vis-à-vis de l'information n'est pas fondée sur la théorie. Le travail empirique n'a pas produit de solides fondations théoriques, et l'étude de la satisfaction vis-à-vis de l'information a été marquée par l'ambiguïté conceptuelle. Bien qu'une définition universelle de la satisfaction des patients ne soit peut-être ni possible ni souhaitable, il convient de clarifier les concepts afin de contribuer à l'élaboration de théories qui puissent guider l'étude de la satisfaction des patients vis-à-vis de l'information .

Le travail empirique continu peut servir de base à l'élaboration de modèles théoriques visant à améliorer la compréhension à la fois des déterminants et des résultats de la satisfaction vis-à-vis de l'information. La recherche à ce jour témoigne de la complexe interdépendance entre les divers facteurs liés aux patients, aux fournisseurs et à l'information elle-même qui détermine la satisfaction des patients et/ou des proches vis-à-vis de l'information. La nature multifactorielle de la satisfaction vis-à-vis de l'information requiert que l'on axe la recherche sur la compréhension des diverses relations entre et au sein des divers facteurs et déterminants. Par exemple, il convient d'examiner plus profondément l'influence médiatrice du style de recherche d'information du patient sur ses attentes en matière d'information et sur ses préférences relativement à la quantité et au type d'information. De plus, les façons dont ces variables liées aux patients influent sur la quantité et le type d'information, de même que sur le choix du moment (variables liées à l'information) constituent un autre domaine d'étude important. Bien que plusieurs déterminants aient été étudiés, de nombreux autres facteurs propres à la population atteinte du cancer demeurent des inconnues, comme par exemple la fatigue attentionnelle, le locus de contrôle et le déni de la réalité. Jusqu'à présent, la recherche portant sur l'impact, sur les résultats, de la satisfaction vis-à-vis de l'information est modeste. Une étude a établi que la satisfaction visà-vis de la quantité de l'information augmentait la qualité de vie des patients (Annunziata et al., 1998). Vu l'importance accordée à la qualité de vie par la population atteinte du cancer, il convient d'étudier plus à fond la relation entre la satisfaction vis-à-vis de l'information et la qualité de vie.

Nombre d'études étaient marquées par des lacunes d'ordre méthodologique. La plupart des études portant sur la satisfaction étaient de nature quantitative et elles adoptaient des formats de type papiercrayon. Un certain nombre d'études n'ont pas rapporté les données psychométriques dérivées des questionnaires, tandis que d'autres n'ont pas même obtenu ces données (voir le résumé présenté au tableau 1). Lorsqu'on mesure la satisfaction générale, on observe que des niveaux élevés d'insatisfaction vis-à-vis de l'information peuvent être occultés par de hauts niveaux de satisfaction vis-à-vis de certains aspects des soins (Abramowitz et al., 1987; Steptoe et al., 1991).

Les devis quantitatifs étaient grandement majoritaires dans le domaine des études de la satisfaction vis-à-vis de l'information, allant de descriptives à expérimentales. L'interdépendance des facteurs liés aux patients, aux fournisseurs et à l'information elle-même a rendu difficile le contrôle des variables parasites dans la recherche, et a conduit à des résultats confus et non concluants. Il est essentiel d'adopter des approches méthodologiques plus rigoureuses, et ce de façon régulière. De plus, l'utilisation exclusive de méthodes transversales et d'études longitudinales pour mesurer la satisfaction en fonction du temps au cours d'une seule phase de l'expérience du cancer fait qu'il est difficile de déterminer avec exactitude l'influence de ladite phase sur la satisfaction vis-à-vis de l'information. Il convient de réaliser des études longitudinales afin d'explorer la satisfaction en fonction des besoins d'information qui changent tout au long de l'évolution de la maladie et selon les différents milieux de soins. Il convient également d'isoler d'autres variables telles que le temps et/ou l'appui offert par la famille, les amis et l'équipe de soins, afin d'étudier la relation entre ces variables et la satisfaction des patients vis-à-vis de l'information. Les études qualitatives font défaut, et pourtant elles sont essentielles si l'on souhaite parvenir à une compréhension approfondie de la satisfaction des patients et des proches vis-à-vis de l'information qu'ils reçoivent.

En général, nous avons observé que l'échantillonnage constituait un point fort des études examinées, malgré certaines lacunes au niveau des méthodes d'échantillonnage. Plus particulièrement, nombre d'études descriptives portant sur les facteurs liés aux patients ont employé de petits échantillons, ce qui a rendu difficile la détermination des effets, sur la satisfaction, des facteurs liés aux personnes et aux milieux (voir le tableau 1). Dans la grande majorité des cas, les échantillons provenaient à chaque fois d'un seul site, ce qui rend la généralisation des résultats difficile. Il serait utile d'échantillonner les 
patients et les familles séparément afin d'identifier pour chacun de ces groupes les déterminants de la satisfaction vis-à-vis de l'information. On pourrait améliorer la valeur des études à venir en effectuant des échantillonnages aléatoires multicentriques et en incorporant les proches ainsi que la population pédiatrique.

Bien que la recherche actuelle ait permis d'approfondir la compréhension de la satisfaction des patients vis-à-vis de divers types d'information relative au cancer, nous ne savons toujours pas ce qui détermine le caractère adéquat et spécifique de l'enseignement aux patients. Le choix du moment où communiquer l'information (type et quantité) requiert des études plus approfondies, puisque la communication prématurée d'une information excessive non accompagnée des discussions nécessaires peut s'avérer préjudiciable. Il convient à l'avenir de déterminer plus précisément l'influence insaisissable de ces facteurs sur la satisfaction des patients. De nombreuses questions sur la nature de l'information que souhaitent recevoir les patients et les familles, sur le moment où cette information devrait être communiquée, et par qui, demeurent sans réponses. Il se peut qu'une approche qualitative soit plus appropriée pour y répondre.

\section{Répercussions pour la pratique}

Les résultats de cette recension des écrits sur la satisfaction des patients et de leur famille vis-à-vis de l'information relative au cancer comportent des répercussions pour les praticiens. Ces derniers doivent savoir qu'il est possible que les patients et les soignants naturels/conjoints soient très satisfaits des services de soins en général tout en étant moins satisfaits de l'information relative au cancer. Lorsque les patients/soignants naturels/conjoints sont satisfaits de l'information, l'observance du traitement est renforcée et les résultats sont meilleurs. Il ne suffit pas de fournir de l'information aux patients et à leurs proches pour qu'ils en soient satisfaits (Jones et al., 1998). Lorsqu'il s'agit de promouvoir la satisfaction des patients/proches vis-à-vis de l'information qu'ils reçoivent, les infirmières doivent tenir compte de leurs propres qualités en tant que fournisseurs d'information, des caractéristiques des patients/proches et de la nature de l'information elle-même.

Les infirmières doivent évaluer les attentes et préférences des patients en matière d'information de même que leur style de recherche d'information afin d'adapter les renseignements aux besoins particuliers de ces derniers. Les patients qui recherchent activement de l'information préfèrent généralement des renseignements relatifs à la maladie et au traitement, et ont des attentes élevées par rapport à l'information qu'ils reçoivent. Il convient d'adopter pour ces patients une approche d'enseignement différente de celle dont on se sert pour les patients qui ont des attentes peu élevées, qui veulent très peu d'information et qui préfèrent des renseignements d'ordre psychosocial. En comprenant les attentes, les préférences et les styles de recherche d'information des patients et des proches, les infirmières seront plus en mesure de personnaliser la quantité et le type d'information qu'elles fournissent, de même que le choix du moment où la fournir. Il importe que les infirmières évaluent les préférences particulières des patients et des soignants naturels/proches afin que leurs besoins en information soient adéquatement comblés.

Les effets secondaires du traitement anticancéreux peuvent engendrer énormément d'anxiété pour les patients et leur famille. Lorsque le niveau d'anxiété augmente, la satisfaction a tendance à chuter. Si l'on souhaite augmenter la satisfaction des patients, il convient de réduire leur anxiété le plus possible, notamment par la gestion efficace des effets secondaires. La mauvaise gestion des effets secondaires du traitement peut mener à l'interruption prématurée de ce dernier. Les effets secondaires survenant et disparaissant tout au long de la période de traitement, il incombe aux infirmières de fournir aux patients une information adéquate afin de les aider à maîtriser les effets secondaires sans toutefois ajouter à leur anxiété. Les cliniciens doivent être disposés à modifier leur façon de fournir l'information relative au cancer au fur et à mesure qu'évoluent le traitement et par là-même, les besoins d'information de leurs patients.

Les fournisseurs de soins de santé doivent également être conscients qu'ils peuvent eux-mêmes influer sur la satisfaction des patients vis-à-vis de l'information. L'accessibilité de l'infirmière est un facteur de satisfaction vis-à-vis de l'information. Il est important que les praticiens soient conscients de leur volonté de partager l'information et de leur ouverture aux questions. Les infirmières doivent tenir compte du style particulier du patient en matière d'information lorsqu'elles décident d'adopter une approche de partage d'information plus ou moins ouverte. Une infirmière qui a tendance à être ouverte dans le partage de l'information devra réduire son débit de partage pour un patient qui préfère en savoir le moins possible. Inversement, une infirmière qui a tendance à ne pas divulguer beaucoup d'information devra s'efforcer d'être plus ouverte pour les patients qui souhaitent plus de renseignements.

Les recherches à ce jour reflètent l'approche ouverte et multidisciplinaire employée auprès de la population atteinte du cancer, une approche très appréciée par les patients et par leur famille. Toutefois, il est possible que la nature multidisciplinaire des soins aux personnes atteintes du cancer mène à la communication d'information contradictoire. La recherche a révélé l'existence d'une corrélation entre l'information contradictoire et l'insatisfaction. Les praticiens peuvent rehausser la satisfaction des patients vis-à-vis de l'information relative au cancer de plusieurs façons: 1) en étant conscients des incohérences dans l'information; 2) en encourageant la communication entre eux et avec les patients et les familles; et 3) en communiquant l'information avec empathie et compassion.

Les praticiens peuvent choisir parmi plusieurs modes de communication qui semblent tous être également propices à la promotion de la satisfaction des patients. Les praticiens doivent s'assurer d'offrir des renseignements aussi pertinents que possible à la situation du patient et de sa famille. Les praticiens ne devraient pas avoir recours exclusivement à l'information écrite telle que les feuillets et livrets, puisque ces modes de communication ont tendance à fournir de l'information de nature plus générale. Bien que le matériel écrit standard soit un bon point de départ, il est important de réaliser en plus une évaluation des besoins d'apprentissage de chaque patient et de personnaliser les renseignements en fonction de cette évaluation. La recherche indique que l'utilisation des bandes sonores a tendance à augmenter la satisfaction des patients. Les praticiens pourraient enregistrer des séances d'information sur bandes sonores pour ensuite mettre ces bandes à la disposition des clients et des proches. Il se peut que ceux-ci se sentent submergés par l'information en plus d'être en proie à énormément d'anxiété. La bande sonore leur donnera l'occasion d'écouter et d'absorber des renseignements qu'ils n'auront peut-être pas captés lors de la séance d'information (Ong et al., 2000).

\section{Conclusion}

L'intérêt accru des chercheurs pour la question de la satisfaction des patients vis-à-vis de l'information relative au cancer a mené à plus de lucidité dans ce domaine. La recherche en est rendue au point où l'élaboration de théories sera bientôt possible. La recherche à ce jour a identifié de nombreux facteurs liés aux patients, aux fournisseurs d'information et à l'information elle-même, facteurs qui déterminent la satisfaction des patients vis-à-vis de l'information relative au cancer. L'étude des résultats de la satisfaction vis-à-vis de l'enseignement au patient en est à ses débuts. Il existe, au sein de la recherche, diverses lacunes qui doivent être comblées. Toutefois, le corpus de recherche actuel constitue un fondement à partir duquel on peut orienter la prestation des soins aux personnes atteintes du cancer.

Les références se trouvent à la page 174 . 
Tableau 1: Synthèse des écrits empiriques sur la satisfaction des patients/proches vis-à-vis de l'information relative au cancer Facteurs liés aux patients et aux proches

\begin{tabular}{|c|c|c|c|c|c|c|}
\hline Auteur(s) & Année & Devis & Échantillonnage & $\mathbf{N}$ & $\begin{array}{l}\text { Variables de } \\
\text { la satisfaction }\end{array}$ & Outil(s) de mesure de la satisfaction \\
\hline $\begin{array}{l}\text { Lundberg } \\
\text { et Trichorb }\end{array}$ & 2001 & $\begin{array}{l}\text { Quantitif } \\
\text { Descriptif }\end{array}$ & $\begin{array}{l}\text { Patients atteints de } \\
\text { cancer recevant une } \\
\text { radiothérapie. } \\
\text { Convenance }\end{array}$ & 200 & $\begin{array}{l}\text { Satisfaction } \\
\text { vis-à-vis de } \\
\text { l'enseignement } \\
\text { et du soutien. }\end{array}$ & $\begin{array}{l}\text { Questionnaire sur l'expérience du patient } \\
\text { comportant } 4 \text { parties élaboré pour l'étude. } \\
\text { Satisfaction mesurée au moyen d'une échelle } \\
\text { de type Likert en } 5 \text { points. }\end{array}$ \\
\hline Ong et al. & 2000 & $\begin{array}{l}\text { Quantitatif } \\
\text { Randomisé } \\
\text { À double insu }\end{array}$ & $\begin{array}{l}\text { Patients atteints de } \\
\text { divers de cancer. } \\
\text { Aléatoire }\end{array}$ & \begin{tabular}{|l|}
201 \\
(105 groupe \\
expéri- \\
mental \\
96 groupe \\
témoin)
\end{tabular} & $\begin{array}{l}\text { Satisfaction with } \\
\text { vis-à-vis de } \\
\text { l'information } \\
\text { reçue. }\end{array}$ & $\begin{array}{l}\text { Questionnaire sur la satisfaction des patients } \\
\text { et Questionnaire sur la satisfaction } \\
\text { des patients-III. } \\
\text { Échelles analogues visuelles. } \\
\text { Fidélité et validité non abordées } \\
\text { dans l'article. }\end{array}$ \\
\hline Aucoin-Gallant & 1999 & $\begin{array}{l}\text { Quantitatif et } \\
\text { qualitatif } \\
\text { Descriptif }\end{array}$ & $\begin{array}{l}\text { Soignantes naturelles } \\
\text { de sujets masculins } \\
\text { atteints de cancer } \\
\text { recevant un traitement } \\
\text { de chimiothérapie ou } \\
\text { radiothérapie. } \\
\text { Convenance }\end{array}$ & 30 & $\begin{array}{l}\text { Satisfaction } \\
\text { vis-à-vis des } \\
\text { besoins } \\
\text { d'apprentissage. }\end{array}$ & $\begin{array}{l}\text { Échelle de satisfaction en matière de } \\
\text { besoins d'apprentissage (1995) - échelle de } \\
\text { type Likert en } 5 \text { points. } \\
\text { A établi la validité du contenu. } \\
\text { Homogénéité et stabilité: } \\
0,80 \text { et } 0,84 \text { respectivement. }\end{array}$ \\
\hline Fitch et al. & 1999 & $\begin{array}{l}\text { Quantitatif } \\
\text { Enquête }\end{array}$ & $\begin{array}{l}\text { Hommes atteints du } \\
\text { cancer de la prostate } \\
\text { ayant suivi un type } \\
\text { de traitement. } \\
\text { Convenance }\end{array}$ & 621 & $\begin{array}{l}\text { Satisfaction } \\
\text { vis-à-vis du } \\
\text { type } \\
\text { d'information } \\
\text { fourni. }\end{array}$ & $\begin{array}{l}\text { Instrument d'enquête à } 52 \text { questions conçu } \\
\text { pour l'étude par les membres de l'équipe de } \\
\text { recherche. Douze semblent mesurer la } \\
\text { satisfaction. } \\
\text { Fidélité et validité non abordées dans l'article. }\end{array}$ \\
\hline Jones et al. & 1999 & $\begin{array}{l}\text { Quantitatif } \\
\text { Essai } \\
\text { randomisé } \\
\text { prospectif } \\
\text { sur séries } \\
\text { temporelles } \\
\text { auprès de } 3 \\
\text { groupes }\end{array}$ & $\begin{array}{l}\text { Patients atteints de } \\
\text { cancer du sein, du col } \\
\text { utérin, de la prostate } \\
\text { et du larynx. } \\
\text { Aléatoire }\end{array}$ & 525 & $\begin{array}{l}\text { Satisfaction } \\
\text { vis-à-vis de } \\
\text { l'information. }\end{array}$ & $\begin{array}{l}\text { Score relatif à la satisfaction calculé à partir } \\
\text { de } 7 \text { attributs présentés dans un } \\
\text { questionnaire. } \\
\text { Fidélité et validité non } \\
\text { abordées dans l'article. }\end{array}$ \\
\hline Kutner et al. & 1999 & $\begin{array}{l}\text { Quantitatif } \\
\text { Essai } \\
\text { randomisé à } \\
2 \text { groupes }\end{array}$ & $\begin{array}{l}\text { Patients ambulatoires } \\
\text { ayant divers diagnostics } \\
\text { de cancer et devant } \\
\text { subir de nouvelles } \\
\text { visites. } \\
\text { Aléatoire }\end{array}$ & 282 & $\begin{array}{l}\text { Satisfaction } \\
\text { vis-à-vis des } \\
\text { explications } \\
\text { des médecins. }\end{array}$ & $\begin{array}{l}\text { L'équipe a élaboré un questionnaire dont } \\
9 \text { questions abordaient la satisfaction au } \\
\text { moyen d'une échelle de type Likert en } \\
5 \text { points. Coefficient alpha } 0,72 \text { pour la } \\
\text { sous-échelle de relation et } 0,65 \text { pour la } \\
\text { sous-échelle de demande. } \\
\text { Validité non abordée dans l'article. }\end{array}$ \\
\hline $\begin{array}{l}\text { Northouse } \\
\text { et al. }\end{array}$ & 1999 & $\begin{array}{l}\text { Qualitatif } \\
\text { Descriptif }\end{array}$ & $\begin{array}{l}\text { Patients atteints du } \\
\text { cancer du côlon. } \\
\text { Convenance }\end{array}$ & $\begin{array}{l}30 \text { patients } \\
\text { et } \\
\text { conjoint(e)s }\end{array}$ & $\begin{array}{l}\text { Satisfaction } \\
\text { vis-à-vis de } \\
\text { l'information } \\
\text { reçue }\end{array}$ & $\begin{array}{l}\text { Entrevue semi-dirigée comportant la question } \\
\text { "Repensez à l'expérience: dans quelle } \\
\text { mesure étiez-vous satisfait(e) de } \\
\text { l'information fournie?" } \\
\text { Validité apparente établie. }\end{array}$ \\
\hline Butow et al. & 1998 & $\begin{array}{l}\text { Quantitatif } \\
\text { Exploratoire }\end{array}$ & $\begin{array}{l}\text { Patients recevant une } \\
\text { chimiothérapie ou } \\
\text { venant de la } \\
\text { terminer. } \\
\text { Aléatoire }\end{array}$ & 24-phase I & $\begin{array}{l}\text { Satisfaction et } \\
\text { préférence des } \\
\text { patients vis-à-vis } \\
\text { de livrets } \\
\text { d'information et } \\
\text { utilisation de } \\
\text { ces derniers. }\end{array}$ & $\begin{array}{l}\text { Phase I: } 6 \text { questions avec échelle Likert } \\
\text { Cohérence interne } 0,64-0,70 \text {; Corrélations } \\
\text { item-total } 0,27-0,87 \\
\text { Phase II: Même échelle que dans la phase I } \\
\text { avec } 2 \text { questions de plus. } \\
\text { Cohérence interne } 0,86 \text {; Corrélations item- } \\
\text { total } 0,40-0,91 \text { avec une moyenne de } 0,74 \text {. }\end{array}$ \\
\hline Jones et al. & 1998 & $\begin{array}{l}\text { Quantitatif } \\
\text { Enquête }\end{array}$ & $\begin{array}{l}\text { Patients atteints de } \\
\text { cancer du sein, du col } \\
\text { utérin, de la prostate et } \\
\text { du larynx sur le point } \\
\text { de recevoir une } \\
\text { radiothérapie } \\
\text { Aléatoire }\end{array}$ & 525 & $\begin{array}{l}\text { Satisfaction } \\
\text { vis-à-vis de } \\
\text { l'information } \\
\text { reçue }\end{array}$ & $\begin{array}{l}\text { Questionnaire (non décrit dans l'article). } \\
\text { Fidélité et validité non } \\
\text { abordées dans l'article. }\end{array}$ \\
\hline
\end{tabular}


Tableau 1: Synthèse des écrits empiriques sur la satisfaction des patients/proches vis-à-vis de l'information relative au cancer Facteurs liés aux patients et aux proches

\begin{tabular}{|c|c|c|c|c|c|c|}
\hline Auteur(s) & Année & Devis & Échantillonnage & $\mathbf{N}$ & $\begin{array}{l}\text { Variables de } \\
\text { la satisfaction }\end{array}$ & Outil(s) de mesure de la satisfaction \\
\hline $\begin{array}{l}\text { Sitzia et } \\
\text { Wood }\end{array}$ & $1998 b$ & $\begin{array}{l}\text { Quantitatif } \\
\text { et qualitatif } \\
\text { Descriptif, } \\
\text { exploratoire }\end{array}$ & $\begin{array}{l}\text { Patients atteints de } \\
\text { divers types de cancer } \\
\text { et recevant une } \\
\text { chimiothérapie. } \\
\text { Aléatoire }\end{array}$ & 173 & $\begin{array}{l}\text { Satisfaction } \\
\text { vis-à-vis de } \\
\text { l'information } \\
\text { relative à la } \\
\text { chimiothérapie, de } \\
\text { l'explication des } \\
\text { procédures et } \\
\text { du traitement, de } \\
\text { l'information sur } \\
\text { regimens, les effets } \\
\text { secondaires, des } \\
\text { protocoles de } \\
\text { chimio, du } \\
\text { pronostic, des } \\
\text { progrès. }\end{array}$ & $\begin{array}{l}\text { Questionnaire de Worthing sur la satisfaction } \\
\text { vis-à-vis de la chimiothérapie. Questionnaire } \\
\text { d'autoévaluation de } 47 \text { questions, dont } 27 \\
\text { portent sur la satisfaction, utilisant des } \\
\text { échelles analogiques visuelles ou une } \\
\text { échelle d'appréciation qualitative en } \\
5 \text { points } \\
\text { Validité et fidélité établies. }\end{array}$ \\
\hline Whelan et al. & 1998 & $\begin{array}{l}\text { Quantitatif } \\
\text { Devis } \\
\text { avant/après }\end{array}$ & $\begin{array}{l}\text { Patients nouvellement } \\
\text { diagnostiqués de } \\
\text { divers types de cancer. } \\
\text { Aléatoire }\end{array}$ & 300 & $\begin{array}{l}\text { Satisfaction } \\
\text { vis-à-vis d'une } \\
\text { chemise de } \\
\text { documentation } \\
\text { personnalisée. }\end{array}$ & $\begin{array}{l}\text { Questionnaire mis au point par l'équipe. } \\
\text { Validité et fidélité non abordées } \\
\text { dans l'article. }\end{array}$ \\
\hline Butow et al. & 1997 & $\begin{array}{l}\text { Quantitatif } \\
\text { Descriptif, } \\
\text { exploratoire }\end{array}$ & $\begin{array}{l}\text { Patients atteints de } \\
\text { divers types de cancer. } \\
\text { Aléatoire }\end{array}$ & 80 & $\begin{array}{l}\text { Satisfaction } \\
\text { vis-à-vis de la } \\
\text { quantité } \\
\text { d'information } \\
\text { fournie et des } \\
\text { techniques de } \\
\text { communication } \\
\text { employées pour } \\
\text { diffuser } \\
\text { l'information }\end{array}$ & $\begin{array}{l}25 \text { questions, échelle Likert. } \\
\text { Fidélité interne } 0,92 . \\
\text { Validité non abordée dans l'article. }\end{array}$ \\
\hline Turner et al. & 1996 & $\begin{array}{l}\text { Quantitatif } \\
\text { Descriptif }\end{array}$ & $\begin{array}{l}\text { Adultes atteints de } \\
\text { la maladie de Hodgkin. } \\
\text { Convenance }\end{array}$ & 165 & $\begin{array}{l}\text { Satisfaction } \\
\text { vis-à-vis de la } \\
\text { quantité } \\
\text { d'information } \\
\text { fournie. }\end{array}$ & $\begin{array}{l}\text { Questionnaire mis au point par l'équipe. } \\
\text { Validité et fidélité non abordées dans l'article }\end{array}$ \\
\hline $\begin{array}{l}\text { Tattersall } \\
\text { et al. }\end{array}$ & 1994 & $\begin{array}{l}\text { Quantitatif } \\
\text { Expérimental }\end{array}$ & $\begin{array}{l}\text { Patients atteints de } \\
\text { divers types de cancer. } \\
\text { Aléatoire }\end{array}$ & 182 & $\begin{array}{l}\text { Satisfaction } \\
\text { vis-à-vis des } \\
\text { aides à la } \\
\text { communication } \\
\text { et utilisation de } \\
\text { ces dernières. }\end{array}$ & $\begin{array}{l}8 \text { questions obtenues à partir d'une analyse } \\
\text { de contenu de commentaires de patients. } \\
\text { Utilisation d'une échelle linéaire analogique } \\
\text { en } 10 \text {-points. } \\
\text { Validité et fidélité non } \\
\text { abordées dans l'article. }\end{array}$ \\
\hline $\begin{array}{l}\text { Derman } \\
\text { \& Serbest }\end{array}$ & 1993 & $\begin{array}{l}\text { Quantitatif } \\
\text { Descriptif }\end{array}$ & $\begin{array}{l}\text { Patients atteints de } \\
\text { divers types de cancer } \\
\text { et recevant un } \\
\text { traitement. } \\
\text { Convenance }\end{array}$ & 45 & $\begin{array}{l}\text { Satisfaction } \\
\text { vis-à-vis de } \\
\text { l'information } \\
\text { fournie. }\end{array}$ & $\begin{array}{l}\text { Entrevue semi-dirigée } \\
\text { comportant } 45 \text { questions. } \\
\text { Validité et fidélité non abordées } \\
\text { dans l'article. }\end{array}$ \\
\hline Dunn et al. & 1993 & $\begin{array}{l}\text { Quantitatif } \\
\text { Essai } \\
\text { randomisé, } \\
3 \text { groupes }\end{array}$ & $\begin{array}{l}\text { Patients atteints de } \\
\text { divers types de cancer } \\
\text { assistant à leur } \\
\text { première consultation } \\
\text { avec un(e) oncologue } \\
\text { médical(e). } \\
\text { Aléatoire }\end{array}$ & 142 & $\begin{array}{l}\text { Satisfaction } \\
\text { vis-à-vis du mode } \\
\text { de diffusion de } \\
\text { l'information } \\
\text { (bande sonore). } \\
\text { Satisfaction } \\
\text { vis-à-vis de la } \\
\text { quantité } \\
\text { d'information } \\
\text { reçue }\end{array}$ & $\begin{array}{l}22 \text { questions tirées du questionnaire } \\
\text { de Roter, Korsch et al. } \\
\text { Validité et fidélité non abordées } \\
\text { dans l'article. }\end{array}$ \\
\hline
\end{tabular}


Tableau 1: Synthèse des écrits empiriques sur la satisfaction des patients/proches vis-à-vis de l'information relative au cancer Facteurs liés aux patients et aux proches

\begin{tabular}{|c|c|c|c|c|c|c|}
\hline Auteur(s) & Année & Devis & Échantillonnage & $\mathbf{N}$ & $\begin{array}{l}\text { Variables de } \\
\text { la satisfaction }\end{array}$ & Outil(s) de mesure de la satisfaction \\
\hline Steptoe et al. & 1991 & $\begin{array}{l}\text { Quantitatif } \\
\text { Exploratoire, } \\
\text { corrélationnel }\end{array}$ & $\begin{array}{l}\text { Patients atteints de } \\
\text { divers types de cancer } \\
\text { admis à l'hôpital pour } \\
\text { évaluation/ traitement. } \\
\text { Convenance }\end{array}$ & 77 & $\begin{array}{l}\text { Satisfaction } \\
\text { vis-à-vis de } \\
\text { l'information } \\
\text { (relative aux } \\
\text { examens, } \\
\text { symptômes et } \\
\text { traitement). } \\
\end{array}$ & $\begin{array}{l}7 \text { échelles en } 5 \text { points. } \\
\text { Fidélité et validité non abordées. }\end{array}$ \\
\hline Derdiarian & 1989 & $\begin{array}{l}\text { Quantitatif } \\
\text { Devis } \\
\text { quasi-expér., } \\
2 \text { groupes }\end{array}$ & $\begin{array}{l}\text { Hommes récemment } \\
\text { diagnostiqués de } \\
\text { divers types de cancer } \\
\text { et conjointes. } \\
\text { Aléatoire }\end{array}$ & $\begin{array}{l}30 \text { patients } \\
\text { et } \\
\text { conjointes, } \\
\text { groupes } \\
\text { expéri- } \\
\text { mental et } \\
\text { témoin }\end{array}$ & $\begin{array}{l}\text { Satisfaction } \\
\text { vis-à-vis de } \\
\text { l'information } \\
\text { reçue }\end{array}$ & $\begin{array}{l}\text { Instruments portant sur la satisfaction des } \\
\text { patients et sur celles des conjointes. } \\
24 \text { questions de type Likert en vue de } \\
\text { mesurer la satisfaction. } \\
\text { Fidélité et validité établies. }\end{array}$ \\
\hline $\begin{array}{l}\text { Sutherland } \\
\text { et al. }\end{array}$ & 1989 & $\begin{array}{l}\text { Quantitatif } \\
\text { Corrélationnel }\end{array}$ & $\begin{array}{l}\text { Patients atteints de } \\
\text { divers types de } \\
\text { cancer recevant une } \\
\text { chimiothérapie et } \\
\text { une radiothérapie. } \\
\text { Convenance }\end{array}$ & 52 & $\begin{array}{l}\text { Satisfaction } \\
\text { vis-à-vis des } \\
\text { détails acquis. }\end{array}$ & $\begin{array}{l}\text { Questionnaire sur la recherche d'information. } \\
18 \text { échelles linéaires analogiques } \\
\text { d'autoévaluation; } 5 \text { d'entre elles explorent la } \\
\text { satisfaction. } \\
\text { Fidélité établie au moyen du coefficient } \\
\text { alpha de Cronbach (valeur non fournie). } \\
\text { Validité examinée au moyen de l'enquête } \\
\text { sur l'opinion relative à la santé. }\end{array}$ \\
\hline $\begin{array}{l}\text { Degner } \\
\& \text { Russell }\end{array}$ & 1988 & $\begin{array}{l}\text { Qualitatif } \\
\text { Descriptif }\end{array}$ & $\begin{array}{l}\text { Patients atteints } \\
\text { de cancer. } \\
\text { Échantillonnage } \\
\text { théorique }\end{array}$ & 60 & $\begin{array}{l}\text { Satisfaction } \\
\text { explorée } \\
\text { indirectement par } \\
\text { le biais de la } \\
\text { préférence en mat } \\
\text { de contrôle pour } \\
\text { la prise de } \\
\text { décision. }\end{array}$ & $\begin{array}{l}8 \text { scénarios écrits } \\
\text { Utilisation d'une procédure de tri de cartes. }\end{array}$ \\
\hline Oberst & 1984 & $\begin{array}{l}\text { Quantitatif } \\
\text { Descriptif, } \\
\text { corrélationnel }\end{array}$ & $\begin{array}{l}\text { Patients ambulatoires } \\
\text { atteints de divers types } \\
\text { de cancer et recevant } \\
\text { une chimiothérapie. } \\
\text { Convenance }\end{array}$ & 20 & $\begin{array}{l}\text { Satisfaction } \\
\text { vis-à-vis de } \\
\text { l'information } \\
\text { relative au } \\
\text { diagnostic et au } \\
\text { traitement, de } \\
\text { l'information sur } \\
\text { les autosoins, la } \\
\text { qualité des soins } \\
\text { médicaux et } \\
\text { infirmiers, et la } \\
\text { mesure dans } \\
\text { laquelle les } \\
\text { attentes liées aux } \\
\text { soins ont été } \\
\text { comblées. }\end{array}$ & $\begin{array}{l}5 \text { échelles analogiques visuelles au sein d'un } \\
\text { questionnaire portaient sur les perceptions et } \\
\text { sur le caractère adéquat des soins. } \\
\text { Fidélité (cohérence interne) non mesurée. } \\
\text { Validité non abordée dans l'article. }\end{array}$ \\
\hline $\begin{array}{l}\text { Reynolds } \\
\text { et al. }\end{array}$ & 1981 & Quantitatif & $\begin{array}{l}\text { Patients atteints de } \\
\text { divers types de cancer. } \\
\text { Aléatoire }\end{array}$ & 67 & $\begin{array}{l}\text { Satisfaction } \\
\text { vis-à-vis de la } \\
\text { quantité } \\
\text { d'information } \\
\text { fournie. }\end{array}$ & $\begin{array}{l}\text { Entrevue dirigée utilisant une échelle } \\
\text { en } 5 \text { points. Le nombre de questions n'est } \\
\text { pas dégagé. } \\
\text { Fidélité et validité non } \\
\text { abordées dans l'article. }\end{array}$ \\
\hline
\end{tabular}


Tableau 1: Synthèse des écrits empiriques sur la satisfaction des patients/proches vis-à-vis de l'information relative au cancer Facteurs liés aux fournisseurs de soins

\begin{tabular}{|c|c|c|c|c|c|c|}
\hline Auteur(s) & Année & Devis & Échantillonnage & $\mathbf{N}$ & $\begin{array}{l}\text { Variables de } \\
\text { la satisfaction }\end{array}$ & Outil(s) de mesure de la satisfaction \\
\hline Aucoin-Gallant & 1999 & \multicolumn{5}{|c|}{ Consulter la section sur les facteurs liés aux patients et aux proches. } \\
\hline Sitzia \& Wood & 1998(b) & \multicolumn{5}{|c|}{ Consulter la section sur les facteurs liés aux patients et aux proches. } \\
\hline Poroch & 1995 & $\begin{array}{l}\text { Quantitatif } \\
\text { Quasi- } \\
\text { expérimental } \\
\text { prospectif }\end{array}$ & $\begin{array}{l}\text { Patients atteints de } \\
\text { divers types de cancer } \\
\text { recevant une } \\
\text { radiothérapie. } \\
\text { Aléatoire }\end{array}$ & $\begin{array}{l}\text { Groupe } \\
\text { standard } \\
\text { pour } \\
\text { l'enseigne- } \\
\text { ment = 25 } \\
\text { Groupe } \\
\text { visé par } \\
\text { l'inter- } \\
\text { vention } \\
=25\end{array}$ & $\begin{array}{l}\text { Satisfaction } \\
\text { vis-à-vis de } \\
\text { l'information et } \\
\text { préférence en } \\
\text { matière } \\
\text { d'opportunité et } \\
\text { présentation de } \\
\text { l'information. }\end{array}$ & $\begin{array}{l}6 \text { sous-échelles adaptées du Questionnaire } \\
\text { de Pienschke sur la satisfaction des patients } \\
\text { (PPQS). } \\
\text { Aucune donnée publiée sur la } \\
\text { validité ou la fidélité du PPQS. }\end{array}$ \\
\hline Tishelman & 1994 & $\begin{array}{l}\text { Quantitatif } \\
\text { Descriptif }\end{array}$ & $\begin{array}{l}\text { Patients atteints de } \\
\text { divers types de cancer. } \\
\text { Aléatoire }\end{array}$ & $\begin{array}{l}1^{\text {re }} \text { entrevue: } \\
46 \\
2^{\mathrm{e}} \text { entrevue: } \\
20\end{array}$ & $\begin{array}{l}\text { Satisfaction } \\
\text { vis-à-vis des soins } \\
\text { infirmiers et de la } \\
\text { communication } \\
\text { d'information. }\end{array}$ & $\begin{array}{l}\text { Entrevue semi-dirigée. } \\
\text { Guide d'entrevue préparé par le chercheur. } \\
\text { Nombre de questions: non précisé. } \\
\text { Fidélité et validité non } \\
\text { abordées dans l'article. }\end{array}$ \\
\hline $\begin{array}{l}\text { Hutchison } \\
\text { et al. }\end{array}$ & 1991 & $\begin{array}{l}\text { Quantitatif } \\
\text { Enquête }\end{array}$ & $\begin{array}{l}\text { Patients atteints de } \\
\text { divers types de cancer. } \\
\text { Convenance }\end{array}$ & 93 & $\begin{array}{l}\text { Satisfaction } \\
\text { vis-à-vis des } \\
\text { soins et des } \\
\text { explications } \\
\text { reçues du } \\
\text { personnel médical } \\
\text { et infirmier. }\end{array}$ & $\begin{array}{l}\text { Enquête élaborée par l'équipe de recherche. } \\
\text { La majorité des questions avaient une échelle } \\
\text { en } 5 \text { points; } 2 \text { en avaient une en } 3 \text { points. } \\
\text { Validité et fidélité non } \\
\text { abordées dans l'article. }\end{array}$ \\
\hline Steptoe et al. & 1991 & \multicolumn{5}{|c|}{ Consulter la section sur les facteurs liés aux patients et aux proches. } \\
\hline Oberst & 1984 & \multicolumn{5}{|c|}{ Consulter la section sur les facteurs liés aux patients et aux proches. } \\
\hline Reynolds et al. & 1981 & \multicolumn{5}{|c|}{ Consulter la section sur les facteurs liés aux patients et aux proches. } \\
\hline Pienschke & 1973 & $\begin{array}{l}\text { Quantitatif } \\
\text { Devis } \\
\text { factoriel } 2 \times 2\end{array}$ & $\begin{array}{l}\text { Patients hospitalisés } \\
\text { subissant une } \\
\text { investigation ou une } \\
\text { chirurgie en raison } \\
\text { d'un cancer. } \\
\text { Aléatoire }\end{array}$ & 32 & $\begin{array}{l}\text { Satisfaction } \\
\text { vis-à-vis de la } \\
\text { démarche de } \\
\text { divulgation de } \\
\text { l'information et } \\
\text { du type, de la } \\
\text { quantité } \\
\text { d'information } \\
\text { fourni(e). }\end{array}$ & $\begin{array}{l}\text { Entrevue-questionnaire auprès des patients } \\
\text { (s'inspirant de Johnson et Thielbar, 1971) } \\
\text { Validité et fidélité non } \\
\text { abordées dans l'article. }\end{array}$ \\
\hline \multicolumn{7}{|c|}{ Facteurs liés à l'information } \\
\hline Auteur(s) & Année & Devis & Échantillonnage & $\mathbf{N}$ & $\begin{array}{l}\text { Variables de } \\
\text { la satisfaction } \\
\end{array}$ & Outil(s) de mesure de la satisfaction \\
\hline D'haese et al. & 2000 & $\begin{array}{l}\text { Quantitatif } \\
\text { Quasi-expér., } \\
\text { Séries } \\
\text { temporelles, } \\
2 \text { groupes }\end{array}$ & $\begin{array}{l}\text { Patients atteints de } \\
\text { divers types de cancer } \\
\text { (tumeurs solides) et } \\
\text { recevant une } \\
\text { radiothérapie. } \\
\text { Aléatoire }\end{array}$ & $\begin{array}{l}68 \\
31 \text { groupe } \\
\text { d'essai } \\
37 \text { groupe } \\
\text { témoin }\end{array}$ & $\begin{array}{l}\text { Satisfaction } \\
\text { vis-à-vis du } \\
\text { moment choisi } \\
\text { pour la } \\
\text { distribution de } \\
\text { l'information } \\
\text { écrite. }\end{array}$ & $\begin{array}{l}\text { Définit la satisfaction et rapporte les scores } \\
\text { rel. à la satisfaction mais ne dit pas comment } \\
\text { ils ont été obtenus. } \\
\text { Validité et fidélité non abordées } \\
\text { dans l'article. }\end{array}$ \\
\hline Ong et al. & 2000 & \multicolumn{5}{|c|}{ Consulter la section sur les facteurs liés aux patients et aux proches. } \\
\hline
\end{tabular}




\begin{tabular}{|c|c|c|c|c|c|c|}
\hline Auteur(s) & Année & Devis & Échantillonnage & $\mathbf{N}$ & $\begin{array}{l}\text { Variables de } \\
\text { la satisfaction }\end{array}$ & Outil(s) de mesure de la satisfaction \\
\hline Porter & 1998 & $\begin{array}{l}\text { Quantitatif } \\
2 \text { groupes, } \\
\text { prospectif, } \\
\text { quasi- } \\
\text { expérimental }\end{array}$ & $\begin{array}{l}\text { Patients adultes atteints } \\
\text { de divers types de } \\
\text { cancer recevant leur } \\
\text { premier traitement de } \\
\text { chimiothérapie. } \\
\text { Aléatoire }\end{array}$ & 240 & $\begin{array}{l}\text { Satisfaction } \\
\text { vis-à-vis des rôles } \\
\text { des infirmières en } \\
\text { oncologie sur le } \\
\text { plan de la } \\
\text { de la profession, } \\
\text { de l'enseignement } \\
\text { et de la confiance } \\
\text { inspirée. }\end{array}$ & $\begin{array}{l}\text { Échelle de satisfaction des patients } \\
\text { (Risser, 1975). Fidélité alpha }=0,92 . \\
\text { Validité non abordée dans l'article. }\end{array}$ \\
\hline Whelan et al. & 1998 & \multicolumn{5}{|c|}{ Consulter la section sur les facteurs liés aux patients et aux proches. } \\
\hline Butow et al. & 1998 & \multicolumn{5}{|c|}{ Consulter la section sur les facteurs liés aux patients et aux proches. } \\
\hline Butow et al. & 1997 & \multicolumn{5}{|c|}{ Consulter la section sur les facteurs liés aux patients et aux proches. } \\
\hline $\begin{array}{l}\text { van Wersch } \\
\text { et al. }\end{array}$ & 1997 & $\begin{array}{l}\text { Quantitatif } \\
2 \text { groupes, } \\
\text { prospectif, } \\
\text { expérimental }\end{array}$ & $\begin{array}{l}\text { Patients atteints de } \\
\text { cancers des voies } \\
\text { aérodigestives } \\
\text { supérieures. } \\
\text { Aléatoire }\end{array}$ & $\begin{array}{l}\text { Groupe } \\
\text { d'essai } \\
\text { Patients } \\
=60 \\
\text { Profess- } \\
\text { ionnels de } \\
\text { la santé } \\
=59 \\
\text { Groupe } \\
\text { témoin } \\
\text { Patients } \\
=39 \\
\text { Profess- } \\
\text { ionnels de } \\
\text { la santé } \\
=35\end{array}$ & $\begin{array}{l}\text { Fonctionnement } \\
\text { psychosocial. } \\
\text { Soutien relatif } \\
\text { aux problèmes } \\
\text { psychosociaux }\end{array}$ & $\begin{array}{l}\text { Questionnaire élaboré par l'équipe de } \\
\text { de recherche. } \\
\text { Validité et fidélité non } \\
\text { abordées dans l'article. }\end{array}$ \\
\hline Hagopian & 1996 & $\begin{array}{l}\text { Quantitatif } \\
\text { groupe témoin } \\
\text { uniquement } \\
\text { en post-test }\end{array}$ & $\begin{array}{l}\text { Patients atteints de } \\
\text { divers types de cancer } \\
\text { recevant une } \\
\text { radiothérapie. } \\
\text { Aléatoire }\end{array}$ & 75 & $\begin{array}{l}\text { Satisfaction } \\
\text { vis-à-vis du mode } \\
\text { de communication } \\
\text { de l'information } \\
\text { (bande audio). }\end{array}$ & $\begin{array}{l}\text { Enquête à échelle de type Likert en } 5 \text { points. } \\
6 \text { des sous-échelles adaptées du } \\
\text { Questionnaire de Pienschke sur la } \\
\text { satisfaction des patients. } \\
\text { Validité et fidélité non } \\
\text { abordées dans l'article. }\end{array}$ \\
\hline Vetto et al. & 1996 & $\begin{array}{l}\text { Quantitatif } \\
\text { Enquête }\end{array}$ & $\begin{array}{l}\text { Femmes présentant } \\
\text { des affections } \\
\text { bénignes du sein. } \\
\text { Aléatoire }\end{array}$ & 130 & $\begin{array}{l}\text { Satisfaction } \\
\text { vis-à-vis du } \\
\text { dépliant. }\end{array}$ & $\begin{array}{l}\text { Enquête renfermant } 4 \text { questions sur la } \\
\text { satisfaction. Échelle de Likert en } 10 \text { points. } \\
\text { Validité et fidélité non } \\
\text { abordées dans l'article. }\end{array}$ \\
\hline Poroch & 1995 & \multicolumn{5}{|c|}{ Consulter la section sur les facteurs liés aux fournisseurs de soins. } \\
\hline Tattersall et al. & 1994 & \multicolumn{5}{|c|}{ Consulter la section sur les facteurs liés aux patients et aux proches. } \\
\hline Dunn et al. & 1993 & \multicolumn{5}{|c|}{ Consulter la section sur les facteurs liés aux patients et aux proches. } \\
\hline Derdiarian & 1989 & \multicolumn{5}{|c|}{ Consulter la section sur les facteurs liés aux patients et aux proches. } \\
\hline Reynolds et al. & 1981 & \multicolumn{5}{|c|}{ Consulter la section sur les facteurs liés aux patients et aux proches. } \\
\hline
\end{tabular}

\section{Références}

Abramowitz, S., Coté, A., \& Berry, E. (1987). Analyzing patient satisfaction: A multianalytic approach. Quality Review Bulletin, 13, 122-130.

Annunziata, M., Foladore, S., Magri, M., Crivellari, D., Feltrin, A., Bidoll, E., \& Veronesi, A. (1998). Does the information level of cancer patients correlate with quality of life? A prospective study. Tumori, 84, 619-623.

Aucoin-Gallant, G. (1999). La description des besoins d'apprentissage et du degré d'insatisfaction de la soignante naturelle. Revue canadienne de soins infirmiers en oncologie, 9, 165-170.
Burish, T.G., \& Tope, D.M. (1992). Psychological techniques for controlling the adverse side-effects of cancer chemotherapy: Findings from a decade of research. Journal of Pain and Symptom Management, 7, 287-301.

Butow, P., Maclean, M., Dunn, S., Tattersall, M., \& Boyer, M. (1997). The dynamics of change: Cancer patients' preferences for information, involvement and support. Annals of Oncology, 8, 857-863. 
Butow, P., Brindle, E., McConnell, D., Boakes, R., \& Tattersall, M. (1998). Information booklets about cancer: Factors influencing patient satisfaction and utilisation. Patient Education and Counselling, 33, 129-141.

Carter, W.B., Inui, T.S., Kukull, W.A., \& Haigh, V.H. (1982). Outcomebased doctor-patient interaction analysis: II. Identifying effective provider and patient behaviour. Medical Care, 20, 550-566.

Cassileth, B.R., Zupkis, R.V., Sutton-Smith, K., \& March, V. (1980). Information and participation preferences among cancer patients. Annals of Internal Medicine, 112, 832-836.

Degner, L., \& Russell, C. (1988). Preferences for treatment control among adults with cancer. Research in Nursing \& Health, 11, 367-374.

Derdiarian, A.K. (1986). Information needs of recently diagnosed cancer patients. Nursing Research, 35, 276-281.

Derdiarian, A.K. (1987). Informational needs of recently diagnosed cancer patients: Part II. Method and description. Cancer Nursing, 10, 156-163.

Derdiarian, A. (1989). Effects of information on recently diagnosed cancer patients' and spouses' satisfaction with care. Cancer Nursing, 12, 285-292.

Derman, U., \& Serbest, P. (1993). Cancer patients' awareness of disease and satisfaction with services: The influence of their general education level. Journal of Cancer Education, 8, 141-144.

D'haese, S., Vinh-Hung, V., Bijdekerke, P., Spinnoy, M., Lochie, N., De Beukeleer, M. et al. (2000). The effect of timing of the provision of information on anxiety and satisfaction of cancer patients receiving radiotherapy. Journal of Cancer Education, 15(4), 223-227.

Dunn, S., Butow, P., Tattersall, M., Jones, Q., Sheldon, J., Taylor, J., \& Sumich, M. (1993). General information tapes inhibit recall of the cancer consultation. Journal of Clinical Oncology, 11, 2279-2285.

Fitch, M., Johnson, B., Gray, R., \& Franssen, E. (1999). L'impact du cancer de la prostate du point de vue des survivants: répercussions pour les soins infirmiers en oncologie. Revue canadienne de soins infirmiers en oncologie, 9, 29-34.

Green, M., Coupland, V., \& Kitzinger, J. (1990). Expectations, experiences and psychological outcomes of childbirth: A prospective study of 825 women. Birth, 17, 15-24.

Hagopian, G. (1996). The effects of informational audiotapes on knowledge and self-care behaviours of patients undergoing radiation therapy. Oncology Nursing Forum, 23, 697-700.

Hall, J.A., Roter, D.L., \& Rand, C.S. (1981). Communication of affect between patient and physician. Journal of Health and Social Behaviour, 22, 18-30.

Hutchison, G., Addington-Hall, J., Bower, M., Austen, M., \& Coombes, C. (1991). European Journal of Cancer Care, 1, 15-18.

Jones, R., Pearson, J., McGregor, S., Cawsey, A., Barrett, A., Craig, N., et al. (1998). Randomized trial of personalized computer based information for cancer patients. British Medical Journal, 319, 1241-1247.

Jones, R., Pearson, J., McGregor, S., Harper Gilmour, W., Atkinson, J., Barrett, A., et al. (1999). Cross sectional survey of patients' satisfaction with information about cancer. British Medical Journal, 319(6), 1247-1248

Kutner, J., Foehner, K., \& Steiner, J. (1999). Evaluation of the impact of a pre-visit questionnaire for addressing cancer patients' information needs. Journal of Cancer Education, 14, 248-253.

Ley, P. (1982). Satisfaction, compliance, and communication. British Journal of Clinical Psychology, 21, 241-254.

Lundberg, P., \& Trichorb, K. (2001). Thai Buddhist patients with cancer undergoing radiation therapy. Cancer Nursing, 24(6), 469-475.

Miller, S. (1987). Monitoring and blunting: Validation of a questionnaire to assess styles of information seeking under threat. Journal of Personality and Social Psychology, 52, 345-353.

Northouse, L.L., Schafer, J., Tipton, J., \& Metivier, L. (1999). The concerns of patients and spouses after the diagnosis of colon cancer: A qualitative analysis. Journal of Wound, Ostomy, and Continence Nursing, 26, 8-17.
Oberst, M. (1984). Patients' perceptions of care: Measurement of quality and satisfaction. Cancer, 10, 2366-2375.

Ong, L.M., Visser, M.R., Lammes, J., van der Velden, B.C., Kuenen, B.C., \& de Haes, J.D. (2000). Effect of providing cancer patients with the audiotaped initial consultation on satisfaction, recall, and quality of life: A randomized, double-blind study. Journal of Clinical Oncology, 18(16), 3052-3060.

Pienschke, D. (1973). Guardedness or openness on the cancer unit. Nursing Research, 22, 484-490.

Poroch, D. (1995). The effect of preparatory patient education on the anxiety and satisfaction of cancer patients receiving radiation therapy. Cancer Nursing, 18, 206-214.

Porter, H.B. (1998). Efficacité et efficience de l'enseignement sur le cancer dispensé par des infirmières. Revue canadienne de soins infirmiers en oncologie, 8, 235-240.

Rees, C.E., \& Bath, P.A. (2000). Exploring the information flow: Partners of women with breast cancer, patients, and health care professionals. Oncology Nursing Forum, 27(8), 1267-1275.

Reynolds, P.M., Byrne, M.J., Sanson-Fisher, R.W., Poole, A.D., \& Harker, J. (1981). Cancer and communication: Information-giving in an oncology clinic. British Medical Journal, 282, 1449-1451.

Richardson, J.L., Marks, G., \& Levine, A. (1988). The influence of symptoms of disease and side effects of treatment on compliance with cancer therapy. Journal of Clinical Oncology, 6, 1746-1751.

Risser, N. (1975). Development of an instrument to measure patient satisfaction with nurses and nursing care in primary care settings. Nursing Research, 24, 45-52.

Sitzia, J., \& Wood, N. (1998a). Patient satisfaction with cancer chemotherapy nursing: A review of the literature. International Journal of Nursing Studies, 35, 1-12.

Sitzia, J., \& Wood, N. (1998b). Study of patient satisfaction with chemotherapy nursing care. European Journal of Oncology Nursing, 2, 142-153.

Steptoe, A., Sutcliffe, I., Allen, B., \& Coombes, C. (1991). Satisfaction with communication, medical knowledge, and coping style in patients with metastatic cancer. Social Science \& Medicine, 32, 627-632.

Sutherland, H.J., Llwellyn-Thomas, H.A., Lockwood, G.A., Tritchler, D.L., \& Till, J.E. (1989). Cancer patients: Their desire for information and participation in treatment decisions. Journal of the Royal Society of Medicine, 82, 260-263.

Tattersall, M., Butow, P., Griffin, A., \& Dunn, S. (1994). The takehome message: Patients prefer consultation audiotapes to summary letters. Journal of Clinical Oncology, 12, 1305-1311.

Tishelman, C. (1994). Cancer patients' hopes and expectations of nursing practice in Stockholm. Scandinavian Journal of Caring Science, 8, 213-222.

Turner, S., Maher, E.J., Young, T., Young, J., \& Hudson, G.V. (1996). What are the information priorities for cancer patients involved in treatment decisions? An experienced surrogate study in Hodgkin's disease. British Journal of Cancer, 73, 222-227.

van Wersch, A., de Boer, M.F., der Does, E., de Jong, P., Knegt, P., Meewvis, C.A., et al. (1997). Continuity of information in cancer care: Evaluation of a logbook. Patient Education and Counselling, 31, 223-236.

Vetto, J., Dubois, P., \& Perez Vetto, I. (1996). The impact of distribution of a patient-education pamphlet in a multidisciplinary breast clinic. Journal of Cancer Education, 11, 148-152.

Waitzkin, H. (1984). Doctor-patient communication: Clinical implications of social scientific research. Journal of the American Medical Association, 252, 2441-2446.

Whelan, T.J., Rath, D., Willan, A., Neimanis, M., Czukar, D., \& Levine, M. (1998). Evaluation of a patient folder to improve the dissemination of written information materials for cancer patients. Cancer, 83, 1620-1625. 